\title{
A Comparison of the Methods used for Selecting Singular values in Image Compression using SVD
}

\author{
Sahar Khalid Ahmed \\ Department of Computer Engineering, \\ College of Engineering, University of Mosul, \\ Mosul, Iraq
}

\begin{abstract}
In this paper, an image compression using singular value decomposition (SVD) transform is presented. The SVD decomposes the image into two eigenvector matrices and a one singular value diagonal matrix. The compression is achieved by selecting some singular values and their associated eigenvectors. The proper selection of the retained singular values is the critical issues in image compression based SVD transform. The SVD transform is applied to the entire image, and also the image is divided into blocks with the SVD applied to each block. The objective of this paper is to study and discuss the methods used to select the singular values that achieve an acceptable image quality with a reduced size.
\end{abstract}

\section{General Terms}

Image processing

\section{Keywords}

Singular Value Decomposition, Image Compression, SVD

\section{INTRODUCTION}

In our lives, enormous amounts of digital images are stored, processed, and transmitted digitally. These amounts of images require a large storage space and a wide bandwidth during transmission. The need arose for finding a way for representing images with a reduced amount of data, this reduction affects the quality of images. Image compression is the field concerned with reducing image size while maintaining an acceptable image quality[1]. The redundant data is the key factor for achieving image compression. Three basic redundant data can be identified and processed. Coding redundancy refers to coding the gray levels of an image using more code symbols than necessary. Interpixel redundancy is the case when the value of any given pixel can be reasonably predicted from the neighboring value pixel. Psychovisual redundancy depends on the fact that the human eye response is different for visual information so some information can be eliminated without significantly affecting the quality of the image. Removing or reducing one or more of these redundancies achieves image compression [2].

In transform coding techniques, the image is mapped into a set of coefficients by using a linear transform, these coefficients are then quantized and coded[2,3]. A large number of these coefficients have a small magnitude which can be quantized or eliminated while keeping a good quality of the reconstructed image[2].

The optimal energy packing efficiency for any given image is obtained by the Singular Value Decomposition (SVD) transform. The use of the SVD in real applications is limited because of the computations needed to compute the eigenvalues and the eigenvectors of large images [4]. In addition, the compression obtained based on SVD is modest because the eigenvalues and its associated eigenvectors must be coded [3]. The DCT, DWT, and SVD transforms are combined in many works[1,3,5,6,7] to achieve better compression results. In most of these works, the image is divided into blocks and a proper transform is applied to each block depending on a predefined criteria. To improve the compression results of the SVD many authors encode the eignvectors using vector quantization[8,9].The compression of the SVD block depends mostly on a proper selection of the retained singular values.

This paper is concerned with the SVD transform and its application in image compression. The methods used for determining the retained singular values are studied and discussed because they represent the most important factor for achieving the best compression results.

\section{RELATED WORKS}

Image Compression is an important field of image processing. Many authors used the SVD combined with other transforms to achieve a good compression results. In [3,5], the image is divided into blocks of $8 * 8$ pixels, and the SVD or DCT transform is applied to each block depends on the standard deviation of that block. Other works [6,7] combine the SVD with the DWT, the image is partitioned into tiles of $64^{*} 64$ pixels, the Average Standard Deviation (ASTD) is computed for each tile depends on the standard deviations of $8 * 8$ subblocks of the tile. If the ASTD is high, the SVD is applied on each $8 * 8$ subblock of the tile otherwise DWT is applied to the tile. In [10], the image is divided into background and region of interest (ROI). The SVD is used to compress the ROI and the DWT is used to compress the background. In [11], the SVD is applied to the entire image and the image is reconstructed after ignoring some singular values then the DWT is applied on the reconstructed image. In [1], the updated rank SVD transform is used as a pre-processing step for the Adaptive Set Partition Hierarchical Tree (ASPIHT). The Compression results of the SVD can be improved by encoding the eigenvectors using vector quantization $[8,9]$.

\section{SVD TRANSFORM}

A digital image is a matrix of numbers representing the intensity value of each pixel in the image[9]. The purpose of the transformation is to decorrelate the pixels of each sub_block or to collect information as possible into the smallest number of coefficients[2].

The SVD is a transform which decomposes the image into three matrices $(U, S$, and $V$ ), two of these matrices ( $U$ and $V$ ) are orthogonal while $S$ is a diagonal matrix. The $S$ components are the singular values of the input matrix, these components are stored in descending order[12,13]. Assume $A$ is an $\mathrm{m}^{*} \mathrm{n}$ image matrix, the SVD transform decomposes $A$ as follows:

$$
A_{m *_{n}}=U_{m{ }^{*} m} S_{m * n}\left(V_{n * n}\right)^{T}
$$


The rank $(r)$ of the image matrix $A$ is the number of non-zero elements in the diagonal matrix $S$ [12]. Assume the eigenvalues of the matrix $A$ are arranged in descending order $\lambda_{1}>=\lambda_{2}>=\ldots \ldots=\lambda_{\mathrm{n}}=0$ [3]. The diagonal matrix $S$ contains the singular values of the matrix $A$ which is the square root of its eigenvalues [13].

The $\boldsymbol{V}$ columns can be computed from

$$
\left(A^{\prime}-\lambda(n) I\right) v(n)=0 \quad n=1,2, \ldots, r
$$

where, $r$ is the rank of $\mathbf{A}, \mathbf{A}^{\prime}=\mathbf{A}^{\mathrm{T}} \mathbf{A}$.

The $\mathbf{U}$ columns are calculated from

$u(n)=\frac{1}{\sqrt{\lambda(n)}} A v(n) \quad n=1,2, \ldots, r$

The blocks can be reconstructed by keeping the $q$ largest singular values and the corresponding eigenvectors

$$
\hat{A}=\sum_{n=1}^{q} \sqrt{\lambda(n) \mu(n)_{v}^{T}} \quad q \leq r
$$

The square error of the reconstructed blocks is equal to the energy of the discarded eigenvalues

$$
\sum_{m=1}^{r} \sum_{n=1}^{r}|A(m, n)-\hat{\mathrm{A}}(m, n)|^{2}=\sum_{n=q+1}^{r} \lambda(n)
$$

where $\mathbf{A}(m, n)$ is the original pixel of the subblock and $\hat{\mathbf{A}}(m, n)$ is the reconstructed pixel of the subblock.

Thus, the reconstructed energy $(E)$ of the subblock is [3,7]

$$
E=\sum_{n=1}^{q} \lambda(n)
$$

\section{SVD TRANSFORM AND IMAGE COMPRESSION}

Image compression based on the SVD transform utilizes the psycovisual idea. The low singular values can be ignored without significantly decreasing the visual nature of the image[12]. When the SVD is applied to the image matrix $A$, three matrices are obtained. Two matrices $(U$ and $V)$ contain the eigenvectors, and the third matrix $(S)$ is a diagonal containing the singular values of the matrix $A$, arranged in a descending order. The number of nonzero elements on the diagonal of the matrix $S$ represents the rank $(r)$ of the input matrix $A$ [8]. Compression is achieved by selecting $q$ singular values such that $q<r$ and the associated eigenvectors. The selected value of $q$ is the critical factor for achieving an acceptable image quality with a reduced image size. There are many methods for selecting the $q$, among these methods are:

\section{Selecting a constant value of $q$.}

2.Using a specified Mean Square Error (MSE) for the image or (sub_image) to determine the value of $q$ [7]. A MSE can be computed as in [5].

$M S E=\frac{1}{m * n} \sum_{n=q+1}^{r} \lambda(n)$

The singular values are discarded starting from lowest singular value and the $M S E$ is computed each time a singular value is discarded until a predefined value of $M S E$ is reached.

3.Setting a predefined percentage of the sum of singular values [1]. In this work a percentage of the discarded singular value is selected, the ignoring process also started from the lowest singular value.

\section{RESULTS}

In this section, The SVD algorithm is applied to (Peppers, Car) images of size $512 * 512$ with 256 gray levels, shown in Figure (1). The SVD is applied in two ways, the first is applying the SVD on the entire image, and the second way is dividing the image into blocks of size $8 * 8$ and the SVD is applied to each block. Different methods are used for determining the retained singular values. The results are compared using the following measurements.

1.The quality of the reconstructed image is measured by a peak signal to noise ratio (PSNR). The PSNR can be defined in an $\mathrm{N}^{*} \mathrm{~N}$ reconstructed image as in [14]:

$P S N R=10 \log _{10} \frac{(L-1)^{2}}{\frac{1}{N^{2}} \sum_{r=\overline{0}}^{N} ! \sum_{c=0}^{N-1}[A(r, c)-\hat{A}(r, c)]^{2}} d B(8)$

Where :

$L:$ Number of gray levels.

$A(r, c)$ :The original image.

$\hat{A}(r, c):$ The reconstructed image.

2. Compression Ratio $(C R)$ is used to measure the amount of compression. The compression ratio is computed as in [13].

$$
C R=\frac{N * N}{(q *(N+N+1))}
$$

Where, $q$ is the number of the retained singular values.

3.Average of the retained singular values. This measurement is used when the image is divided into $\mathrm{n}$ blocks, and the SVD is applied to each block.

Average $(q)=\frac{\sum_{i=1}^{n} q_{i}}{n}$

Where, $q_{i}$ is the number of the retained singular values in block i.

The compression ratio ( $C R$ ) and PSNR of applying SVD on the entire image are tabulated as follows, in Table (1) constant $q$ is used, Table(2) uses a specified MSE for determining $q$, and Table(3) specifies a percentage of the discarded singular values for determining $q$. As It can be seen from the results, the constant selection of the of $q$ is not a good method because the optimal value is different depending on the image used. The best method is to compute the $q$ value depending on a specified MSE of the image. Figure (2) shows the results of using the three methods mentioned.

The results (PSNR, average q, and maximum q) of applying SVD on each $8 * 8$ block of the divided image are tabulated as follows. Table(4) shows the result when $q$ is constant for all blocks, Table(5) uses a predefined MSE for determining $q$ of 
each block, and Table(6) uses a predefined percentage of the discarded singular values for determining a block's $q$. Figure (3) and Figure(4) shows the results of the three methods. As it can be seen from the results choosing a constant value of $q$ is impractical because the amount of information is different from block to block. Figure (5) shows that the MSE method is better than a percentage of the discarded singular value method for selecting the retained singular values.

Table 1. PSNR and $C R$ when SVD applied on entire image, $q$ is constant.

\begin{tabular}{|l|l|l|l|}
\hline Image name & Constant $\boldsymbol{q}$ & $\boldsymbol{P S N R}$ & $\boldsymbol{C R}$ \\
\hline Car512 & 10 & 25.6889 & 25.5750 \\
\hline Car512 & 20 & 29.9011 & 12.7875 \\
\hline Car512 & 50 & 39.0361 & 5.1150 \\
\hline Car512 & 100 & 47.2217 & 2.5575 \\
\hline Car512 & 130 & 50.7210 & 1.9673 \\
\hline Peppers512 & 10 & 21.4459 & 25.5750 \\
\hline Peppers512 & 20 & 24.9654 & 12.7875 \\
\hline Peppers512 & 50 & 30.4720 & 5.1150 \\
\hline Peppers512 & 100 & 34.9638 & 2.5575 \\
\hline Peppers512 & 130 & 36.8838 & 1.9673 \\
\hline
\end{tabular}

Table 2. PSNR and $C R$ when SVD is applied on the entire image, $M S E$ is used to determine $q$.

\begin{tabular}{|l|l|l|l|l|}
\hline $\begin{array}{l}\text { Image } \\
\text { name }\end{array}$ & $\boldsymbol{M S E}$ & $\boldsymbol{P S N R}$ & $\begin{array}{l}\text { obtained } \\
\boldsymbol{q}\end{array}$ & $\boldsymbol{C R}$ \\
\hline Car512 & 5 & 41.1352 & 60 & 4.2625 \\
\hline Car512 & 10 & 38.2810 & 47 & 5.4415 \\
\hline Car512 & 15 & 36.4426 & 40 & 6.3638 \\
\hline Car512 & 20 & 35.2912 & 36 & 7.1042 \\
\hline Peppers512 & 5 & 41.1266 & 207 & 1.2355 \\
\hline Peppers512 & 10 & 38.1504 & 152 & 1.6826 \\
\hline Peppers512 & 15 & 36.3934 & 122 & 2.0963 \\
\hline Peppers512 & 20 & 35.1692 & 103 & 2.4830 \\
\hline
\end{tabular}

Table 3: PSNR and $C R$ when SVD applied on entire image, percentage of the discarded eigenvalues is used to determine $q$.

\begin{tabular}{|l|l|l|l|l|}
\hline $\begin{array}{l}\text { Image } \\
\text { name }\end{array}$ & $\begin{array}{l}\text { discarded } \\
\text { singular } \\
\text { values }\end{array}$ & PSNR & $\begin{array}{l}\text { The } \\
\text { obtained } \\
\boldsymbol{q}\end{array}$ & $\boldsymbol{C R}$ \\
\hline Car512 & $5 \%$ & 45.5272 & 87 & 2.9397 \\
\hline Car512 & $10 \%$ & 39.5078 & 52 & 4.9183 \\
\hline Car512 & $15 \%$ & 35.2912 & 36 & 7.1042 \\
\hline Car512 & $20 \%$ & 32.3048 & 27 & 9.4722 \\
\hline Peppers512 & $5 \%$ & 43.4834 & 250 & 1.0230 \\
\hline
\end{tabular}

\begin{tabular}{|l|l|l|l|l|}
\hline Peppers512 & $10 \%$ & 38.5353 & 159 & 1.6085 \\
\hline Peppers512 & $15 \%$ & 35.3039 & 105 & 2.4357 \\
\hline Peppers512 & $20 \%$ & 32.7024 & 71 & 3.6021 \\
\hline
\end{tabular}

Table 4: PSNR when SVD applied on $8 * 8$ blocks of the image, $q$ is constant.

\begin{tabular}{|l|l|l|}
\hline Image name & Constant $\boldsymbol{q}$ & PSNR \\
\hline Car512 & 1 & 35.8582 \\
\hline Car512 & 2 & 46.9680 \\
\hline Peppers512 & 1 & 30.3920 \\
\hline Peppers512 & 2 & 36.0612 \\
\hline
\end{tabular}

Table 5: PSNR , average $q$, and maximum $q$ value. SVD is applied on $8 * 8$ blocks of the image, $M S E$ is used to determine $q$.

\begin{tabular}{|l|l|l|l|l|}
\hline $\begin{array}{l}\text { Image } \\
\text { name }\end{array}$ & $\boldsymbol{M S E}$ & $\boldsymbol{P S N R}$ & $\begin{array}{l}\text { Average } \\
\boldsymbol{q}\end{array}$ & Max. $\boldsymbol{q}$ \\
\hline Car512 & 5 & 45.5072 & 1.2788 & 3 \\
\hline Car512 & 10 & 44.2811 & 1.1841 & 3 \\
\hline Car512 & 15 & 43.6121 & 1.1482 & 3 \\
\hline Car512 & 20 & 43.0726 & 1.1250 & 3 \\
\hline Peppers512 & 5 & 42.7388 & 2.9143 & 7 \\
\hline Peppers512 & 7 & 41.3936 & 2.5005 & 7 \\
\hline Peppers512 & 10 & 40.0277 & 2.0942 & 7 \\
\hline Peppers512 & 15 & 38.8151 & 1.7846 & 6 \\
\hline Peppers512 & 20 & 38.0614 & 1.5898 & 6 \\
\hline
\end{tabular}

Table 6: $P S N R$, average $q$, and maximum $q$ value. SVD is applied on $8 * 8$ blocks of the image, a percentage of the discarded singular value is used to determine $q$.

\begin{tabular}{|l|l|l|l|l|}
\hline $\begin{array}{l}\text { Image } \\
\text { name }\end{array}$ & $\begin{array}{l}\text { discarded } \\
\text { singular } \\
\text { values }\end{array}$ & PSNR & $\begin{array}{l}\text { Average } \\
\boldsymbol{q}\end{array}$ & $\begin{array}{l}\text { Max. } \\
\boldsymbol{q}\end{array}$ \\
\hline Car512 & $5 \%$ & 43.5591 & 1.4124 & 3 \\
\hline Car512 & $10 \%$ & 39.8227 & 1.0979 & 3 \\
\hline Car512 & $15 \%$ & 37.3902 & 1.0220 & 2 \\
\hline Car512 & $20 \%$ & 36.4622 & 1.0061 & 2 \\
\hline Peppers512 & $5 \%$ & 40.3794 & 2.7813 & 6 \\
\hline Peppers512 & $7 \%$ & 38.3808 & 2.2551 & 6 \\
\hline Peppers512 & $10 \%$ & 36.3073 & 1.7712 & 5 \\
\hline Peppers512 & $15 \%$ & 34.1136 & 1.388 & 4 \\
\hline Peppers512 & $20 \%$ & 32.7503 & 1.2173 & 4 \\
\hline
\end{tabular}




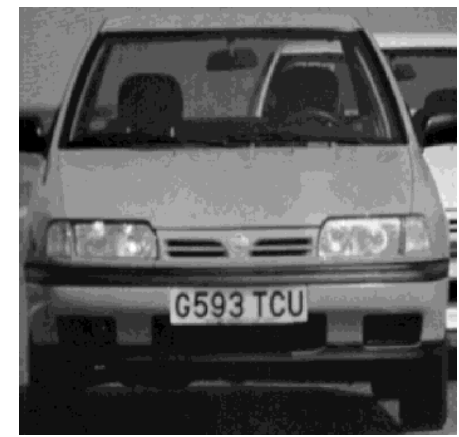

(a) Original Car Image

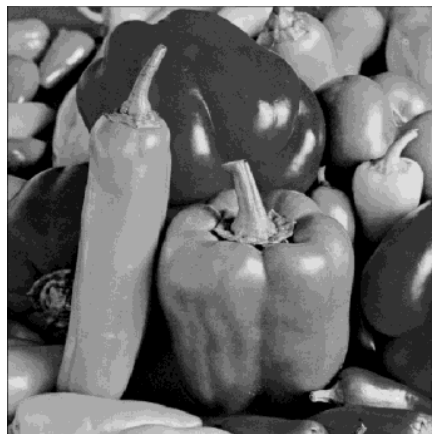

(b) Original Peppers Image

Figure 1: (a) Original car image, (b) Original Peppers image

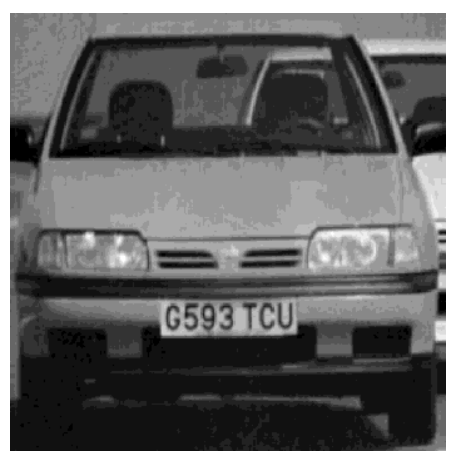

(a) Decoded image, constant $q=50$, PSNR=39.036.

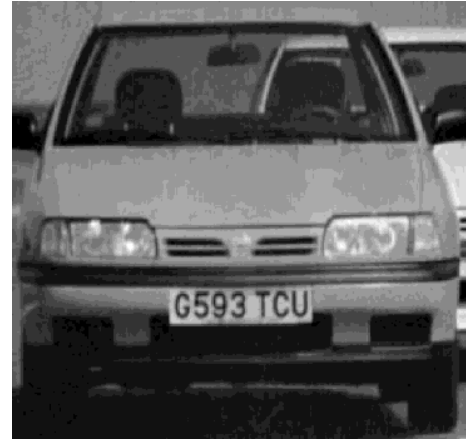

(b) Decoded image, using MSE $=10$. Retained q=47, PSNR=38.281.

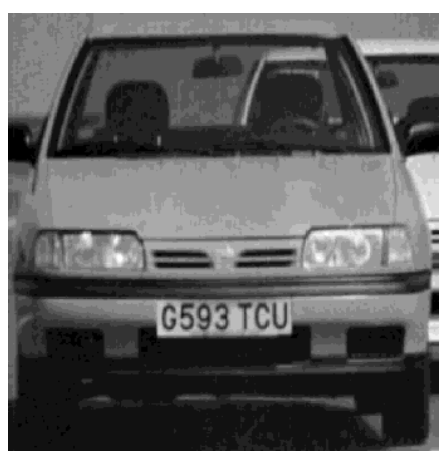

(c) Decoded image, using percentage $=10$. Retained $q=52$, PSNR=39.508.

Figure 2: Decoded Car images. SVD is applied on the entire image

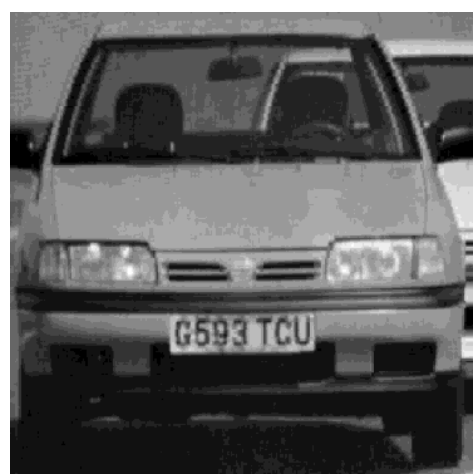

(a)Decoded image, constant $\mathrm{q}=1$, PSNR=35.858.

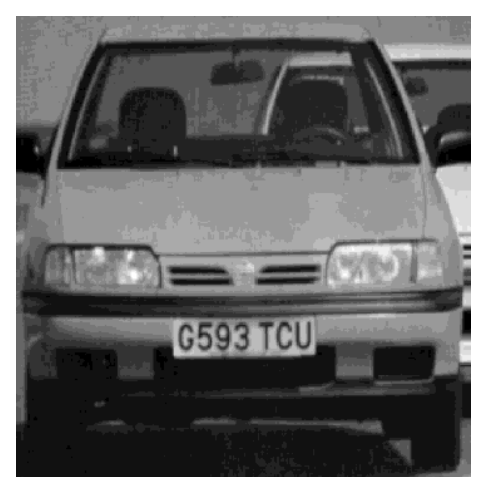

(b)Decoded image, using MSE $=30$. Average $\mathrm{q}=\mathbf{1 . 0 9 6}$, PSNR=42.257.

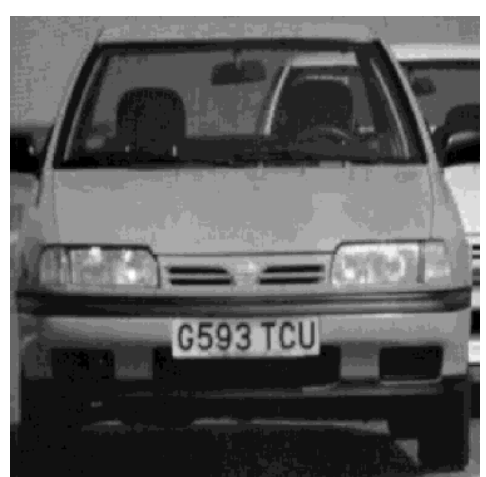

( c ) Decoded image, using percentage $=10$. Average $q=1.098$, PSNR=39.823.

Fiqure 3: Decoded car images. The image is divided into $8 * 8$ blocks, SVD is applied on each block. 


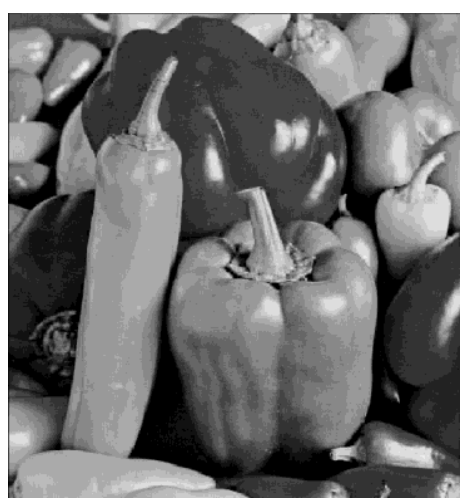

(a)Decoded image, constant $\mathrm{q}=2$, PSNR=36.061.

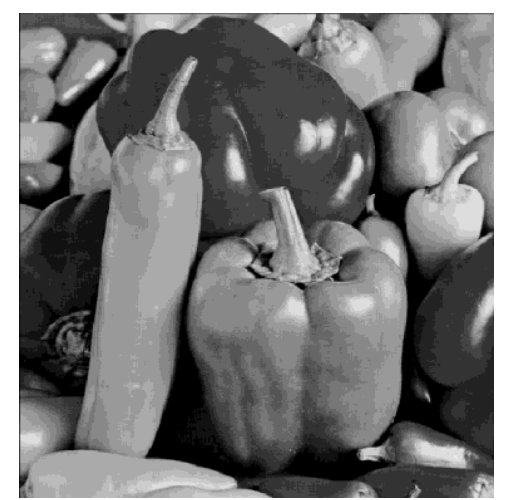

(b)Decoded image, using MSE $=15$. Average $q=1.765$, $P S N R=38.815$.

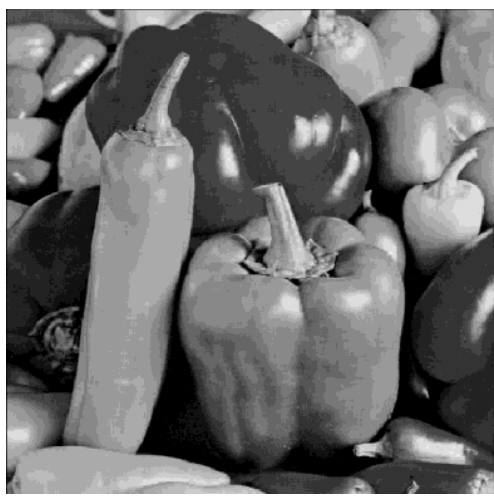

( c) Decoded image, using percentage $=10$. Average $q=1.771$, PSNR=36.307.

Figure 4: Decoded Peppers images. The image is divided into $8 * 8$ blocks, SVD is applied on each block.

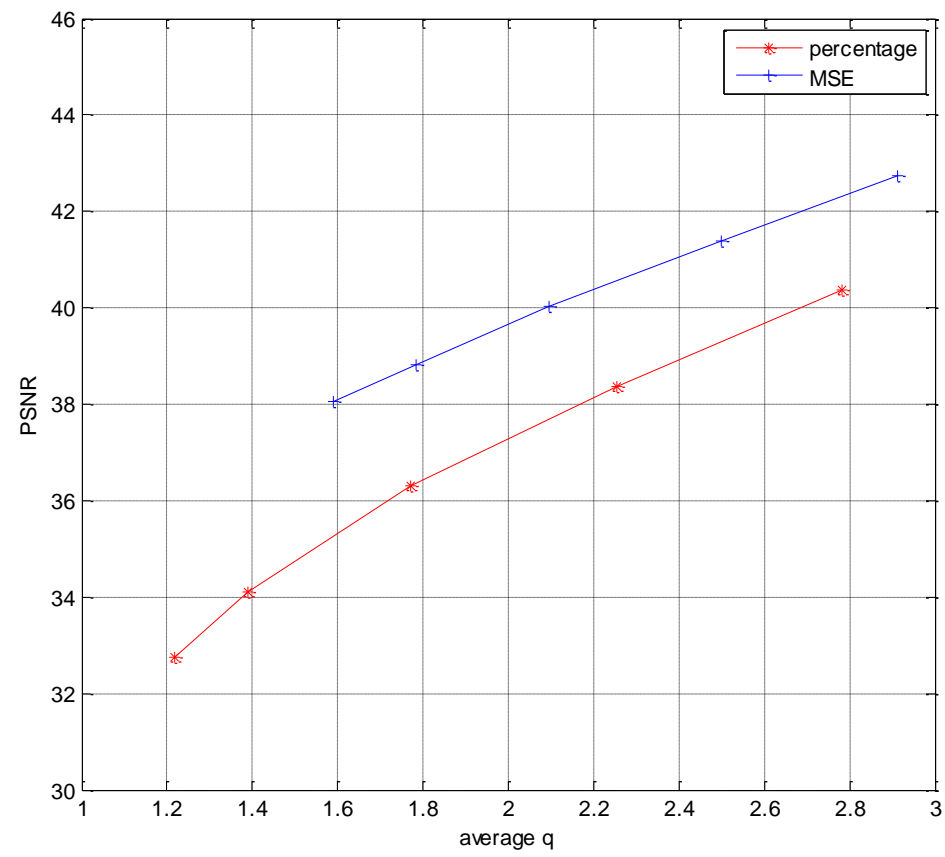

Figure 5:A comparison between the $M S E$ method, and a percentage of the discarded singular value method for $8 * 8$ blocks image "Peppers".

\section{CONCLUSION}

In this paper, the methods used to determine the retained singular values are studied and tested on images. The SVD transform is applied to the entire image and on the blocks of the divided image. Choosing the constant $(q)$ singular values is an impractical method because the optimal value of $q$ varies depending on the nature of the image or block on the divided image. The MSE method which determines the $q$ singular values depending on a predefined MSE of the image or block gave better results than a constant $q$ singular values and a percentage methods.

\section{REFERENCES}

[1] R.Naveen Kumar, B. N. Jagadale and J. S. Bhat" Hybrid Image Compression using Modified Singular Value Decomposition and Adaptive Set Partitioning in Hierarchical Tree", Indian Journal of Science and
Technology, Vol 10(28),

10.17485/ijst/2017/v10i28/101590, July 2017.

[2] R.C. Gonzalez and R.E. Woods, Digital Image Processing. 2nd Edition. Upper Saddle River, NJ: Prentice Hall, 2002.

[3] A. Dapena and S. Ahalt, "A Hybrid DCT-SVD ImageCoding Algorithm," IEEE Trans. CSVT, vol. 12, Feb. 2002, pp.114-121.

[4] A.K. Jain, Fundamentals of Digital Image Processing. Englewood Cliffs, NJ: Prentice-Hall, 1989.

[5] Y. Wongsawat, H. Ochoa, K.R. Rao "A MODIFIED HYBRID DCT-SVD IMAGE-CODING SYSTEM" TECHNO 2004, 2004 IEEE Region 10 Conference, pp:335-338,vol. 1. 
[6] Humberto Ochoa, K.R. Rao"A Low Bit-Rate Hybrid DWT-SVD Image-Coding System (HDWTSVD) for Monochromatic Images", Circuits and systems, 2003,ISCAS'03. Proceedings of the 2003 International synposiun on.

[7] Humberto Ochoa, K.R. Rao"A modified HDWTSVD image coding system" ,Proceedings of the $5^{\text {th }}$ WSEAS international Conference on applications of electrical engineering, march,2006,pp 54-58.

[8] Jar-Ferr Yang, Chjou-hang Lu "Combined Techniques of Singular Value Decomposition and Vector Quantization for Image Coding",IEEE TRANSACTIONS ON IMAGE PROCESSING. VOL 4, NO 8, AUGUST 1995, pp11411145 .

[9] C. S.Goldrick, W. J. Dowling, and A. bury, "Image Coding Using the Singular Value Decomposition and Vector Quantization", in IEE Conf. Pub., 1995, no. 410,pp. 296-300.
[10] Y. He, L. Tong, and M. Jia, "A Compression Image Combining Wavelet Transform With SVD", Computational Problem_Solving (ICCP), 2011 International Conference.

[11] T. Prabakar Joshua, M. Arrivukannamma, and J.G.R. Sathiaseelan, " Lossy Image Compression Using Singular Value Decomposition and Discrete Wavelet Transform", I J C T A, 9(27), 2016, pp. 569-574.

[12] A. M. Rufai, G. Anbarjafari, H. Demirel "Lossy Image Compression Using Singular value Decomposition and Wavelet Difference Reduction", Digital Signal Processing 24(2014)117-123.

[13] S. Kahu, R. Rahate "Image Compression Using Singular Value decomposition", International Journal of Advancements in Research\& Technology, Vol. 2,Issue 8, August-2013, pp 244-248.

[14] Digital Image Processing and Analysis: Human and Computer Application With CVI Ptools, Second Edition, CRC press Taylor\&Francis Group,2010. 